\title{
Corrections
}

\section{Correction: Parvizi et al., Electrical Stimulation of Human Fusiform Face-Selective Regions Distorts Face Perception}

In the article "Electrical Stimulation of Human Fusiform Face-Selective Regions Distorts Face Perception" by Josef Parvizi, Corentin Jacques, Brett L. Foster, Nathan Withoft, Vinitha Rangarajan, Kevin S. Weiner, and Kalanit Grill-Spector, which appeared on pages 14915-14920 of the October 24, 2012 issue, the authors regret misspelling the fourth author's last name. The corrected author line is: Josef Parvizi, Corentin Jacques, Brett L. Foster, Nathan Witthoft, Vinitha Rangarajan, Kevin S. Weiner, and Kalanit Grill-Spector.

DOI: 10.1523/JNEUROSCI.5614-12.2013 